\title{
Altes optimieren, Neues gestalten
}

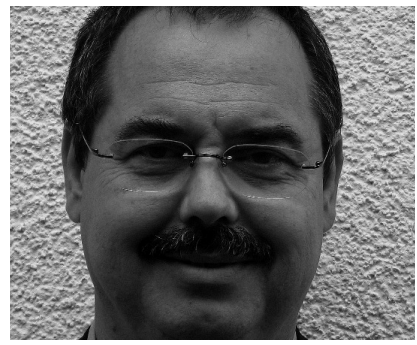

VON PAUL BRANDL

Prof. (FH) Dr. Paul Brandl studierte Wirtschaftspädagogik mit dem Schwerpunkt Organisation an der Universität Linz und promovierte 1985. Er ist heute Professor an der Fachhochschule Oberösterreich in Linz im Studiengang Sozial- und Verwaltungsmanagement.

E-Mail Paul.Brandl@fh-linz.at

\section{Auf der Suche nach Einsparungspotenzialen kommt man an der Optimierung von Prozessen und Strukturen nicht vorbei. Dazu gehört die ständige Weiterentwick- lung der Dienstleistungen entlang den sich verändern- den Bedürfnissen der Kundinnen und Kunden. Wie das funktionieren kann, kann am Beispiel der stationären Altenbetreuung dargestellt werden.}

Derzeit wird das Neudenken und Neugestalten bei sozialen Dienstleistungsorganisationen noch weitgehend vernachlässigt. Würde man insbesondere entlang der »Wertschöpfungskette " (1) die Einführung von (unternehmensübergreifenden) Software-Lösungen sowie der Einführung von neuen Formen der Zusammenarbeit zweier oder mehr Organisationen forcieren (2), so könnte dieser Aspekt der Prozessorganisation zu Einsparungen bei gleichzeitig höherem Kundennutzen führen. Dabei entstehen neue Dienstleistungen, was anhand von drei Beispielen demonstriert werden soll.

\section{Erstes Beispiel:}

\section{Medikamentenversorgung im Alten- und Pflegeheim}

Derzeit ist das »Einschachteln « von Medikamenten in den Kernprozess »Betreuung und Pflege « integriert, wird von diplomiertem Krankenpflegepersonal erledigt und ist sehr zeitaufwendig. Auch Fehlmedikation ist auf diese Weise eine Gefahr; ist es doch eine Arbeit, die häufig in der Nacht erledigt werden muss und oft mit vielen Störungen verbunden ist.

Bei einem funktionalen Ablauf tütet das Personal ein und verständigt beim Fehlen von Medikamenten den Arzt. Der Arzt untersucht, diagnostiziert und verschreibt die erforderlichen Medikamente. Das Rezept wird der Apotheke übermittelt und von dieser ins Altenund Pflegeheim gebracht. Schlimmstenfalls werden die Medikamente unsortiert in einer großen Schachtel beim Portier abgegeben, günstigstenfalls erfolgt eine Lieferung auf die Station gebündelt je Person. Die Apotheke oder das Heim verrechnen die Medikamente. Die Krankenversicherung zahlt die abgerechneten Medikamente, von denen viele meist in angebrochenen Packungen - in jeder Station herumliegen und auch schon mal verloren gehen. Die Krankenkassen stöhnen unter den Kosten.

Beim Einsatz einer »Pillenmaschine « übernimmt im Idealfall die Software die Verständigung des Arztes, dieser trägt seine Diagnose und Therapie in seiner Datenbank ein. Diese gleicht mit der Pflegedokumentation des Pflegeheimes fehlerfrei ab. Von dort holt sich der Apotheker das elektronische Rezept, verpackt mit einem Fast-Dispensing-System die Medikamente so, wie sie am Bett des Bewohners gebraucht werden und verrechnet sowohl mit dem Bewohner als auch mit der Krankenkasse elektronisch die pillengenaue Abgabe der Medikamente.

Dass dieses Modell in eingeschränkter Form auch ohne Informationstechnik kostendeckend funktionieren kann, wurde am Beispiel des Alten- und Pflege- 


\begin{tabular}{|l|l|c|l|c|}
\hline $\begin{array}{l}\text { Häufigkeit } \\
\text { der Tätigkeit }\end{array}$ & Verrechnung - IST-Prozess & $\begin{array}{c}\text { Zeit in } \\
\text { Minuten }\end{array}$ & $\begin{array}{l}\text { Verrechnung - SOLL - } \\
\text { Prozess }\end{array}$ & $\begin{array}{c}\text { Zeit in } \\
\text { Minuten }\end{array}$ \\
\hline $\begin{array}{l}\text { einmalig pro } \\
\text { Klient }\end{array}$ & Klientenstammblatt erstellen & 5 & Klientenstammblatt erstellen & 5 \\
\hline & Stundensatz berechnen & 10 & Stundensatz berechnen & 5 \\
\hline & Summe & $\mathbf{1 5}$ & Summe & $\mathbf{1 0}$ \\
\hline & & & & \\
\hline $\begin{array}{l}\text { monatlich } \\
\text { pro Klient }\end{array}$ & $\begin{array}{l}\text { Verrechenbare Stunden } \\
\text { berechnen }\end{array}$ & 10 & & 2 \\
\hline & Anzahl der Hausbesuche & 5 & & \\
\hline & $\begin{array}{l}\text { Dateneingabe in } \\
\text { Verrechnungsprogramm }\end{array}$ & 5 & $\begin{array}{l}\text { Einspielung der Daten in das } \\
\text { Verrechnungsprogramm }\end{array}$ & \\
\hline & $\begin{array}{l}\text { Erlagschein drucken und } \\
\text { versenden }\end{array}$ & 7 & Abbuchung der Forderung & \\
\hline & $\begin{array}{l}\text { Ausbuchen des Betrages aus } \\
\text { dem Verrechnungsprogramm }\end{array}$ & 5 & & $\mathbf{4}$ \\
\hline & Summe & $\mathbf{3 2}$ & & 5 \\
\hline $\begin{array}{l}\text { jährlich pro } \\
\text { Klient }\end{array}$ & Jahresstatistik erstellen & 15 & Jahresstatistik erstellen & \\
\hline & & & & 19 \\
\hline & Gesamtzeit pro Klient & $\mathbf{6 2}$ & Gesamtzeit pro Klient & \\
\hline
\end{tabular}

Mit der Erfassung von Zeitaufwand und Häufigkeit können die Kostenersparnisse durch neue Prozessabläufe - hier am Beispiel eines Verwaltungsprozesses in einem ambulanten Pflegedienst-eindrucksvoll gezeigt werden.

Quelle: SOZIALwirtschaft 2/2010, Seite 12.

heimes Alland (3) in Österreich untersucht, ebenso die Einsparungsmöglichkeiten in einem Alten- und Pflegeheim (4). Dieses Modell sollte auch auf die mobile Pflege ausgedehnt werden können.

Probleme der Realisierung sind in der Umsetzung bei folgenden Punkten und Zielgruppen zu finden:

- die Mediziner, die sich auf etwa 330 Medikamente beschränken und zunehmend mit IT-Einsatz arbeiten müssen

- die Apotheker, die um die eigenen Einnahmen fürchten, wenn sie keine anderen Beteiligungsmöglichkeiten entwickeln

- die Juristen, die angesetzt werden, um die Realisierung derartiger Projekte im Auftrag von Interessenvertretungen zu verhindern

- das Pflegepersonal, das seine Kompetenzen schwinden sieht und eine Neuorientierung noch nicht als Chance begreift

\section{Zweites Beispiel: Medizinische Standardleistungen im Alten- und Pflegeheime statt im Krankenhaus}

Ausgangspunkt kann die Untersuchung von Andreas Schlecht (5) sein, der anschaulich nachweist, dass beispiels- weise die Behandlung eines Harnwegsinfekts in einem Alten- und Pflegeheim deutlich billiger sein könnte: Er errechnet ein Potenzial für das Alten- und Pflegeheim von etwa 2.000 Euro je Krankheitsfall.

Auch wenn er die nachstehenden Fragen bezüglich der Ausstattung und Qualifizierung nicht berücksichtigt, so untermauert er mit seiner Arbeit die Ergebnisse der Studie von Christian Jagsch, Irmgard Wintgen-Samhaber und Klaus Zapotocky (6). Durch die "ambulante "Behandlung der Heimbewohnerinnen und Heimbewohner im Altenund Pflegeheim konnte erreicht werden, dass nur mehr schwerere Fälle ins Krankenhaus eingewiesen wurden. Er sieht als Mediziner Potenzial für folgende Bereiche: Psychiatrie (Depression, Angst, Wahn, Sucht etc.), Geriatrie und Innere Medizin.

Die medizinische Sinnhaftigkeit dieser Überlegungen wird durch die jüngst veröffentlichte Arbeit von Olivia Kada, Rudolf Likar, Georg Pinter, Karl Cernic, Herbert Janig (7) gestützt. Die Ergebnisse ihrer Untersuchungen hinterfragen kritisch die Sinnhaftigkeit der Transporte von alten Menschen von einem Alten- und Pflegeheim in ein Krankenhaus im derzeitigen Umfang. Die Resultate gehen konform mit der Forderung von Margit Mayr und Markus
Lehner (8) nach Reduzierung der Verlegungen alter, oft multimorbider Menschen in ein Krankenhaus. Auch Heimund Pflegedienstleitungen vermuten, dass sich hier Handlungspotenzial befinden könnte.

Will man an dieser Stelle eine neue Dienstleistung - ein Mittelding von Krankenhaus, Reha-Einrichtung und Pflegeheim - etablieren, so gilt es eine Reihe von Bedenken, Forderungen und Überlegungen lösungsorientiert zu einem Businessplan zu verarbeiten:

- Welche medizinischen Diagnosen eignen sich für eine derartige Dienstleistung?

- Welche Ausstattung und welche pflegerischen Qualifikationen werden benötigt, damit diese qualitativ einwandfrei in einem Alten- und Pflegeheim erbracht werden können? Welche Tagsätze könnten diese Leistungen abdecken?

- Wie muss die (fach-)ärztliche Versorgung der Heimbewohnerinnen und Heimbewohner aussehen, um eine qualitativ einwandfreie Leistung zu garantieren?

- Welche rechtlichen Rahmenbedingungen und Vereinbarungen zwischen Sozial- und Gesundheitsressort müssen dazu geschaffen werden? 
Der Nutzen einer derartigen Dienstleistung wäre einfach zu beschreiben:

- Die durchweg älteren Heimbewohnerinnen und Heimbewohner würden physisch und psychisch weniger belastet.

- Die Alten- und Pflegeheime würden hinsichtlich des Arbeitsbereiches, der Ausstattung und des qualifizierteren Personals aufgewertet.

- Die bereits stark belasteten Krankenversicherungen und die öffentlichen Haushalte (Ressorts Gesundheit und Soziales) würden unterm Strich entlastet, die Alten- und Pflegeheime könnten gleichzeitig mehr Wertschöpfung generieren.

- Die spezialisiert arbeitenden Krankenhäuser würden von einfacheren Fällen von vielfach multimorbiden Patientinnen und Patienten entlastet.

\section{Drittes Beispiel: Die mobile Altenbetreuung und Krankenpflege als Vertriebskanal}

Ausgangspunkt der Überlegungen des Kooperationsprojektes (9) war die Erfahrung mit den Eltern des Verfassers, die zunehmend immobiler wurden und über eine Versorgung mit Lebensmittel und Pflegeprodukten froh gewesen wären. Eine Versorgung durch die mobile Altenbetreuung auch im Bereich der Wäsche und der Pflegeartikel wäre eine willkommene Entlastung für die Angehörigen gewesen. Wir wären auch bereit gewesen, für diese Dienstleistungen extra zu bezahlen. Aus diesen Beweggründen heraus haben wir an der $\mathrm{FH}$
Oberösterreich, Fakultät für Gesundheit und Soziales ein Projekt initiiert, indem wir zusammen mit einem Anbieter von mobiler Altenbetreuung und einer Logistikfirma untersuchen,

- welche Wäscheteile und Pflegeartikel die Klienten einer Region in welchem Ausmaß nachfragen würden und tung, Verrechnung und (elektronischen) Bezahlung muss geklärt sein. Eine Kontrolle muss einfach möglich sein.

- Es bietet sich die Chance, mit dem Vertrieb Geld zu verdienen und so das Pflegeangebot zu stützen.

- Es bedarf einer Einbindung in die bestehende Organisation des Anbieters

\section{"Wertschöpfung entsteht auch durch optimierte Prozesse"}

wie der Ablauf von der Bestellung bis zur Bezahlung sein könnte und

- welche Bedingungen (Schulung der Mitarbeiterinnen und Mitarbeiter, Zwischenlager, Abrechnung etc.) geschaffen werden müssen, damit das Pilotprojekt erfolgreich sein kann.

Abgesehen von der juristischen Abklärung flossen folgende Gedanken und Überlegungen in die Erstellung des Geschäftsmodells ein:

- Die Unterstützung der Angehörigen muss in der Konzeption berücksichtigt werden.

- Es muss in jedem Fall die Wahlfreiheit der Inanspruchnahme gegeben sein.

- Das zu erstellende Businessmodell muss sich rechnen.

- Die Bestellung, Belieferung einschließlich einer möglichen Lagerhal- mobiler Dienstleistungen durch Beteiligung der Mitarbeiterinnen und Mitarbeiter an der Entwicklung dieser Dienstleistung.

- Die bestehende Organisation darf damit nicht eingeschränkt werden. Daher sind Ressourcen und Bearbeitungszeit vorzusehen.

- Die Zahl der zu betreuenden Senioren und Singles wird zunehmen.

- Auch »betreubares « und Betreutes Wohnen wären ein weiteres Potenzial.

- Mobile Altenbetreuung könnte mit dieser neuen Dienstleistung noch attraktiver werden.

Mit Hilfe von Interviews mit den Betreuenden, den Klienten und den Angehörigen hinsichtlich der Abwicklung und der Preisgestaltung werden die Grundlagen für die Erstellung des Ge-

\section{Arbeitsabläufe verbessern, neue Angebote gestalten}

Anbieter sozialer Dienstleistungen kommen durch die demografische Entwicklung und die Folgen wirtschaftlicher Krisenerscheinungen unter ökonomischen Druck. Um die Leistungsqualität auf hohem Niveau halten zu können, müssen die vorhandenen Ressourcen durch ein Optimieren der Prozesse und Strukturen besser genutzt werden. Zudem ist eine innovative Neugestaltung der Dienstleistungen angesagt. Das Buch von Paul Brandl zeigt anhand praktischer Beispiele, wie das Optimieren von Arbeitsabläufen im Bereich der mobilen und stationären Altenpflege realisiert werden kann, und bietet eine theoretische Fundierung dafür. Ein zweiter Fokus liegt auf dem Neugestalten von Dienstleistungen und auf neuen Formen der Zusammenarbeit zwischen bestehenden Einrichtungen.

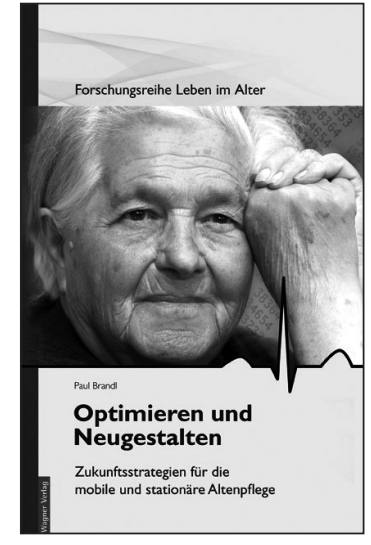

Paul Brandl: Optimieren und Neugestalten. Zukunftsstrategien für die mobile und stationäre Altenpflege. Wagner Verlag, Linz 2010. 144 Seiten. 14,- Euro. ISBN 978-3902330-43-7. 
schäftsmodells zusammengetragen. Eine erste praktische Umsetzung des Pilotprojekts wäre innerhalb von sechs Monaten möglich. Voraussetzung ist allerdings die endgültige Zustimmung des Anbieters von mobilen Pflegedienstleistungen.

\section{Nachlese}

Im ersten Beispiel entsteht eine neue Dienstleistung durch den Einsatz von neuen Technologien und in weiterer Folge von dazu passender Software. Die innovativen und kostensparenden Potenziale der Prozessorganisation sind nur dann zu erschließen, wenn mehrere Organisationen im Hinblick auf den Kundennutzen - hier für die Heimbewohnerinnen und Heimbewohner in Altenund Pflegeheimen - systemübergreifend optimal zusammenarbeiten. Nur dann können zusammen mit neuer Software und Technologie gleichzeitig Vorteile für alle Beteiligten erzielt werden.

Im zweiten Beispiel beruht die neue Dienstleistung aus der konsequenten Erforschung von Schnittstellen zwischen Altenheim und Krankenhaus, die in ei- ner kundenorientierten Gestaltung dieser Angebote enden. Im Zuge dieser Bemühungen entstand die Idee zur Verlagerung des Erbringens von medizinischen Standarddienstleistungen vom Krankenhaus ins Alten- und Pflegeheim.

Im dritten Beispiel entsteht schließlich eine neue Dienstleistung aus der Überlegung, dass die durchwegs älteren Klienten in ihrer Mobilität mehr oder minder eingeschränkt sind und so ein Nutzen daraus erzielt werden kann, in dem die Dienstleistung zum Klienten kommt. Ein Einsatz von mobiler Informationstechnik kann auch hier wirksam unterstützen. Gemeinsam ist allen drei Lösungen eine bessere Prozessorganisation mit erfreulichen Ergebnissen: eine höhere Qualität für den Kunden und weniger Kosten für alle Beteiligten.

\section{Anmerkungen}

(1) Vgl. Paul Brandl, Optimieren und Neugestalten. Zukunftsstrategien für die mobile und stationäre Altenpflege, Wagner Verlag, Linz, 2010.

(2) Vgl. Paul Brandl: a.a.O.
(3) Vgl. Hübler, unv. Praktikumsbericht, Wien, 2009.

(4) Vgl. Ronald Schauer, Medikationssysteme im Alten-/Pflegeheim, unv. Diplomarbeit, Linz, 2009.

(5) Vgl. Andreas Schlecht, Lean Care. Neue wertschätzende und wertschöpfende Dienstleistungen für Alten- und Pflegeheime, unv. Diplomarbeit, Linz, 2009.

(6) Vgl. Christian Jagsch, Irmgard Wintgen-Samhaber, Klaus Zapotocky, Lebensqualität im Seniorenheim. Medizinische, psychotherapeutische und soziologische Aspekte, Linz, 2. überarbeitete Auflage 2006.

(7) Vgl. Olivia Kada, Rudolf Likar, Georg Pinter, Karl Cernic, Herbert Janig, Krankenhaustransporte aus Altenund Pflegeheimen in Kärnten, Feldkirchen, Klagenfurt, 2010.

(8) Vgl. Margit Mayr, Markus Lehner, Stationäre Versorgung älterer Menschen in Oberösterreich. Versorgungsnetzwerke und -prozesse, Linz 2008.

(9) Ein Innovationsprojekt im Rahmen des Masterstudienganges "Services of General Interest « an der FH Oberösterreich, Fakultät für Gesundheit und Soziales im Sommersemester 2010.

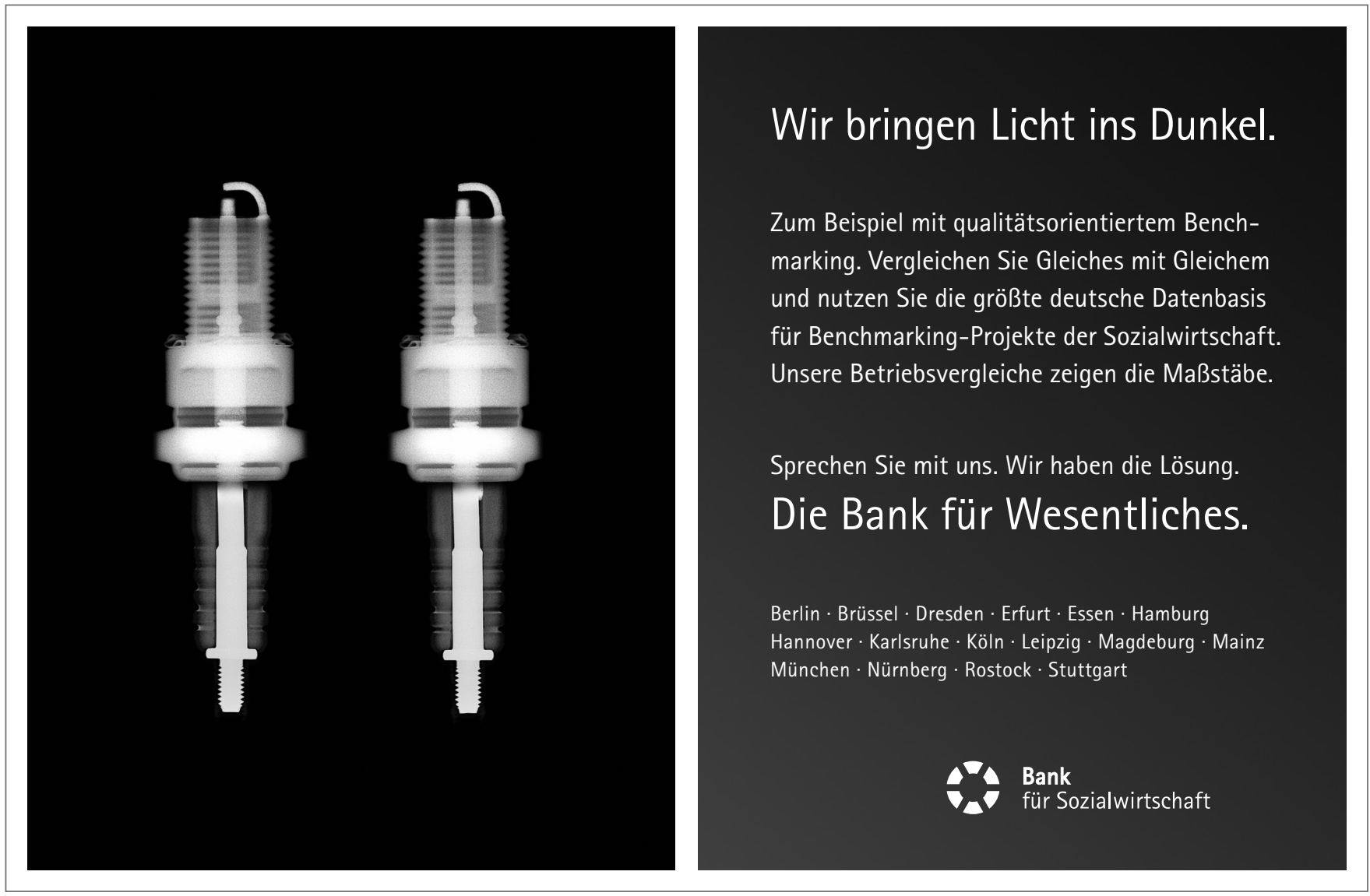

